\title{
Artificial immune recognition system with nonlinear resource allocation method and application to traditional Malay music genre classification
}

\begin{abstract}
Artificial Immune Recognition System (AIRS) has shown an effective performance on several machine learning problems. In this study, the resource allocation method of AIRS was changed with a nonlinear method. This new algorithm, AIRS with nonlinear resource allocation method, was used as a classifier in Traditional Malay Music (TMM) genre classification. Music genre classification has a great important role in music information retrieval systems nowadays. The proposed system consists of three stages: feature extraction, feature selection and finally using proposed algorithm as a classifier. Based on results of conducted experiments, the obtained classification accuracy of proposed system is $88.6 \%$ using 10 fold cross validation for TMM genre classification. The results also show that AIRS with nonlinear allocation method obtains maximum classification accuracy for TMM genre classification.
\end{abstract}

Keyword: Artificial immune system; AIRS; Music genre classification; Nonlinear resource allocation 